\title{
NEW INEQUALITIES FOR ROTOR FRAMES IN HILBERT SPACE
}

\section{K. RAJU PILlai* AND S. PALANiAMMAL}

\begin{abstract}
In this paper, a new identity of the Weyl-Heisenberg frame using a rotation operator has been investigated in Hilbert space. The characterization and significance of Rotor frame inequalities have been discussed by using rotation and translation operators. Also discussed the application of Rotor frames using a rotation operator. Finally, the reconstruction theorem has been investigated for recovers original data. In this work, we would like to highlight that the reconstruction theorem used to obtain the energy of the signal and reconstruct the original signal with eradicated the garbage vector using frame operator in Hilbert space. Today, this technique is very useful in communication systems.
\end{abstract}

Mathematics subject classification (2010): 42C15, 46L99, 47A58.

Keywords and phrases: Hilbert space, frames, Weyl-Heisenberg frame, orthonormal basis, operators.

\section{REFERENCES}

[1] R. J. Duffin, A. C. SchaefFer, A Class of Nonharmonic Fourier Series, Transactions of the Am. Math. Soc., Vol. 72, (1952), 341-366.

[2] K. Raju Pillai, S. Palaniammal, A New identity of Parseval frame through twiddle factor and application in communication systems, Inter. Journal of Appl. Math. Sciences, 10 (80) (2016) pp. 214-218.

[3] I. Daubechies, A. Grossmann, Y. Meyer, Painless nonorthogonal Expansions, Math. phys, 27, (1986), 1271-1283.

[4] K. Raju Pillai, S. Palaniammal, A certain investigation of operator and frame-theory, Jour. of Math.Archive, 10 (2015) pp. 122-128.

[5] P. G. Cas AZZa, Ole Christensen, Perturbation of Operators and Applications to frames Theory, Fourier Anal. Appl, 3, (1997), 543-557.

[6] Radu Balan, Peter Casazza, Dan Edidin, Laura Walters and Eric Weber, On signal reonstruction without phase, Applied and Computational Harmonic Analysis, 20, (2006), 345-356.

[7] K. Raju Pillai, S. Palaniammal, Computational Intelligence and Appli cation of frames Theory in Communication Systems, American Journal of Engineering and Applied Sciences, 8 (4), (2015), 633-637.

[8] Rajendra Bhatia, Modulus of continuity of the matrix absolute Value, J. Pure Appl. Math, 41 (2), (2010), 99-111.

[9] K. Raju Pillai, S. Palaniammal, Reconstruction Theorem for frames in Hilbert Space and application in Communication Systems, Inter. Journal of Applied Engg Research, 10 (80), (2015), 214-218.

[10] Dorin Ervin Dutkay, Deguang Han, Gabriel Picioroaga, Parseval frames for ICC groups, Journal of Functional Analysis, 256, (2009), 3071-3090.

[11] K. Raju Pillai, S. Palaniammal, Certain investigations of $K$-frames in Hilbert space and its application in Cryptography, Inter. Journal of Applied Engineering Research, 11 (1), (2016), 31-35.

[12] K. Raju Pillai, S. Palaniammal, Frames Theory and Application in Digital image Processing, Inter. Journal of L. T. in Engg and Tech, 6 (1) (2015) pp. 61-68.

[13] Guohui Song, ANNE GElB, Approximating the inverse frame operator from localized frames, Appl. Comput. Harmon. Anal, 35, (2013), 94-110.

[14] K. Raju Pillai, S. Palaniammal, Harp frames operator in Hilbert space, Inter. Jour. Pure and Appl. Math, 116 (24) (2017) pp. 131-141. 
[15] P. Balazs, D. T. Stoeva, Representation of the inverse of a frame multiplier, J. Math. Anal. Appl, 422, (2015), 981-994.

[16] K. Raju Pillai, S. Palaniammal, $\alpha$-Phase-Retrieval frame in Hilbert space and its application, Mathematics and Computers in Simulation, 155) (2019) pp. 269-276.

[17] C. EASWARAN NAMBUDIRI AND K. PARTHASARATHY, Generalised Weyl-Heisenberg frame operators, Bull. Sci. math, 136, (2012), 44-53.

[18] K. Raju Pillai, S. Palaniammal, Frames Reconstruction with Noise Reduction in Hilbert space and Application in Communication Systems, Mathematics and Computers in Simulation, 155 (2019) pp. 324-334.

[19] ZHONG-Qi XIAng, New Inequalities for $g$-frames in Hilbert $C^{*}-$ Modules, Journal of Mathematical Inequalities, 10 (3), (2016), 889-897. 